\title{
Classical Representation of a Quantum System at Equilibrium
}

\author{
James W. Dufty*1, + and Sandipan Dutta ${ }^{1,+}$ \\ ${ }^{1}$ Department of Physics, University of Florida, Gainesville, FL 32611
}

(Dated: November 8, 2018)

\begin{abstract}
A quantum system at equilibrium is represented by a corresponding classical system, chosen to reproduce the thermodynamic and structural properties. The objective is to develop a means for exploiting strong coupling classical methods (e.g., MD, integral equations, DFT) to describe quantum systems. The classical system has an effective temperature, local chemical potential, and pair interaction that are defined by requiring equivalence of the grand potential and its functional derivatives with respect to the external and pair potentials for the classical and quantum systems. Practical inversion of this mapping for the classical properties is effected via the hypernetted chain approximation, leading to representations as functionals of the quantum pair correlation function. As an illustration, the parameters of the classical system are determined approximately such that ideal gas and weak coupling RPA limits are preserved.
\end{abstract}

\footnotetext{
* Corresponding author E-mail: Dufty@phys.ufl.edu

${ }^{\dagger}$ Department of Physics, University of Florida, Gainesville, FL 32611

${ }^{\ddagger}$ Electronic address: duttas@phys.ufl.edu
} 


\section{INTRODUCTION}

A simple atomic system in its liquid state is a prototypical strongly coupled system with no small expansion parameters available to simplify calculations. Under conditions where classical mechanics is applicable there are a number of theoretical and computational methods available to address such strong coupling conditions. These include integral equation and density functional methods, and numerical techniques such as molecular dynamics (MD) and Monte Carlo (MC) simulation. For quantum systems there are complementary manybody methods to address the new features of diffraction and exchange or degeneracy effects. Conditions of both quantum degeneracy and strong coupling pose challenging problems with few choices for accurate computation of properties sensitive to both features (e.g., diffusion and path integral Monte Carlo (PIMC) simulation).

One approach to describe such quantum systems is to extend the classical methods in a phenomenological way. For example, MD has been used with effective potentials modified to include short range diffraction effects [1]. A related approach, wave packet MD, uses a similar variant of classical mechanics to define trajectories guiding wave-packets. An interesting new approach is to use classical integral equations for pair correlations modified to include an effective potential and an effective temperature [2]. Quantum effects are imbedded in these effective properties, while strong correlations are generated by the classical form of the integral equation. A surprisingly wide class of quantum systems have been described in this way with considerable success [3].

The objective here is to formalize the exploitation of classical methods by defining a classical statistical mechanics whose equilibrium properties are the same as those for an underlying quantum system. The exact definition is given in the next section, providing the basis for the introduction of practical approximations. One simple realization of electron gas as a classical system is described in the following sections. The emphasis here is on the definition of the mapping and its approximate evaluation. Applications will be described elsewhere. 


\section{DEFINITION OF THE CLASSICAL SYSTEM}

Consider for simplicity a one component system at equilibrium represented in the grand canonical ensemble. The grand potential $\Omega(\beta \mid \mu, \phi)$ is proportional to the pressure and is given by

$$
\Omega(\beta \mid \mu, \phi)=-p(\beta \mid \mu, \phi) V=-\beta^{-1} \ln \sum_{N} \operatorname{Tr}_{N} e^{-\beta\left(K+\Phi-\int d \mathbf{r} \mu(\mathbf{r}) \widehat{n}(\mathbf{r})\right)}
$$

Here $\operatorname{Tr}_{N}$ denotes a trace over properly symmetrized $N$ particle states. The thermodynamics for the system is specified as functions of the inverse temperature $\beta$ and functionals of the local chemical potential $\mu(\mathbf{r}) \equiv \mu-\phi_{\text {ext }}(\mathbf{r})$ (an external potential has been included for generality) and pair potential $\phi$. The Hamiltonian $H=K+\Phi$ contains the kinetic energy $K$ and sum over pair potentials: $\Phi=\frac{1}{2} \sum_{i j} \phi\left(q_{i j}\right)$.

A corresponding classical system is considered with Hamiltonian $H_{c}$ in the same volume $V$ at equilibrium described by the classical grand canonical ensemble, with inverse temperature $\beta_{c}$ and local chemical potential $\mu_{c}(\mathbf{r})=\mu_{c}-\phi_{c, e x t}(\mathbf{r})$. The Hamiltonian has the same form except the potential energy functions $\phi_{c}\left(q_{i j}\right)$ and $\phi_{c, e x t}\left(\mathbf{q}_{i}\right)$ are different. The classical grand potential is defined in terms of these quantities by

$$
\Omega_{c}\left(\beta_{c} \mid \mu_{c}, \phi_{c}\right)=-p_{c}\left(\beta_{c} \mid \mu_{c}, \phi_{c}\right) V=-\frac{1}{\beta_{c}} \ln \sum_{N} \frac{1}{\lambda_{c}^{3 N} N !} \int d \mathbf{q}_{1} . . d \mathbf{q}_{N} e^{-\beta_{c}\left(\Phi_{c}-\int d r \mu_{c}(r) \widehat{n}(r)\right)} .
$$

Here, $\lambda_{c}=\left(2 \pi \beta_{c} \hbar^{2} / m\right)^{1 / 2}$ is the thermal de Broglie wavelength associated with the classical temperature. The integration for the partition function is taken over the $N$ particle configuration space.

The classical system has undefined ingredients: the effective inverse temperature, $\beta_{c}$, the local chemical potential, $\mu_{c}(\mathbf{r})$, and the pair potential for interaction among the particles, $\phi_{c}\left(q_{i j}\right)$. A correspondence between the classical and quantum systems is defined by expressing these quantities as functions or functionals of $\beta, \mu(\mathbf{r})$, and $\phi\left(q_{i j}\right)$. This is accomplished by requiring the numerical equivalence of two independent thermodynamic properties and one structural property. The first two are chosen to be the equivalence of the grand potential and its first functional derivative with respect to the local chemical potential.

$$
\Omega_{c}\left(\beta_{c} \mid \mu_{c}, \phi_{c}\right) \equiv \Omega(\beta \mid \mu, \phi),\left.\left.\quad \frac{\delta \Omega_{c}\left(\beta_{c} \mid \mu_{c}, \phi_{c}\right)}{\delta \mu_{c}(\mathbf{r})}\right|_{\beta_{c}, \phi_{c}} \equiv \frac{\delta \Omega(\beta \mid \mu, \phi)}{\delta \mu(\mathbf{r})}\right|_{\beta, \phi} .
$$


An equivalent form for these conditions can be given in terms of the pressure and density

$$
p_{c}\left(\beta_{c} \mid \mu_{c}, \phi_{c}\right) \equiv p(\beta \mid \mu, \phi), \quad n_{c}\left(\mathbf{r} ; \beta_{c} \mid \mu_{c}, \phi_{c}\right) \equiv n(\mathbf{r} ; \beta \mid \mu, \phi) .
$$

These two relations provide two independent relations between $\beta_{c}, \mu_{c}(\mathbf{r})$ and the physical variables $\beta$ and $\mu(\mathbf{r})$. It remains to have a structural equivalence to relate the pair potential $\phi_{c}\left(q_{i j}\right)$ to $\phi\left(q_{i j}\right)$, which are two particle functions. This is accomplished by equating the functional derivatives of the grand potentials with respect to these pair potentials

$$
\left.\frac{\delta \Omega_{c}\left(\beta_{c} \mid \mu_{c}, \phi_{c}\right)}{\delta \phi_{c}\left(\mathbf{r}, \mathbf{r}^{\prime}\right)}\right|_{\beta_{c}, \mu_{c}}=\left.\frac{\delta \Omega(\beta \mid \mu, \phi)}{\delta \phi\left(\mathbf{r}, \mathbf{r}^{\prime}\right)}\right|_{\beta, \mu} .
$$

An equivalent form for this definition is the equivalence of pair correlation functions

$$
g_{c}\left(\mathbf{r}, \mathbf{r}^{\prime} ; \beta_{c} \mid \mu_{c}, \phi_{c}\right) \equiv g\left(\mathbf{r}, \mathbf{r}^{\prime} ; \beta \mid \mu, \phi\right)
$$

In this way the three equations of (44) and (6) determine, formally, the classical parameters $\beta_{c}, \mu_{c}$, and $\phi_{c}(q)$ as functions of $\beta$, and functionals of $\mu(\mathbf{r})$, and $\phi(q): \beta_{c}=\beta_{c}(\beta \mid \mu, \phi), \quad \mu_{c}=$

$\mu_{c}(\mathbf{r} ; \beta \mid \mu, \phi), \quad \phi_{c}=\phi_{c}\left(\mathbf{r}, \mathbf{r}^{\prime} ; \beta \mid \mu, \phi\right)$. This completes the definition of the thermodynamics of a classical system that is representative of that for a given quantum system. It is also possible to deduce from this a classical density functional theory that is representative of the quantum density functional theory. This will be discussed elsewhere.

\section{INVERSION OF THE MAP}

Exact representations of the classical parameters as functions/functionals of the quantum properties follow from classical density functional theory which has the form

$$
\beta_{c} \mu_{c}(\mathbf{r})=\mathcal{I}\left(\mathbf{r} \mid n_{c}, g_{c}\right), \quad \beta_{c} \phi_{c}\left(\mathbf{r}, \mathbf{r}^{\prime}\right)=\mathcal{J}\left(\mathbf{r}, \mathbf{r}^{\prime} \mid n_{c}, g_{c}\right)
$$

Here $\mathcal{I}(\mathbf{r} \mid \cdot, \cdot)$ and $\mathcal{J}(\mathbf{r} \mid \cdot, \cdot)$ denote formal functionals of the local density and pair correlation function. But according to the definitions (44) and (6) these can be replaced by their corresponding quantum properties to give the desired result

$$
\beta_{c} \mu_{c}(\mathbf{r})=\mathcal{I}(\mathbf{r} \mid n, g), \quad \beta_{c} \phi_{c}\left(\mathbf{r}, \mathbf{r}^{\prime}\right)=\mathcal{J}\left(\mathbf{r}, \mathbf{r}^{\prime} \mid n, g\right)
$$

This provides the formal expressions for the classical local chemical potential and classical pair potential in terms of properties of the quantum system, suitable for the introduction of 

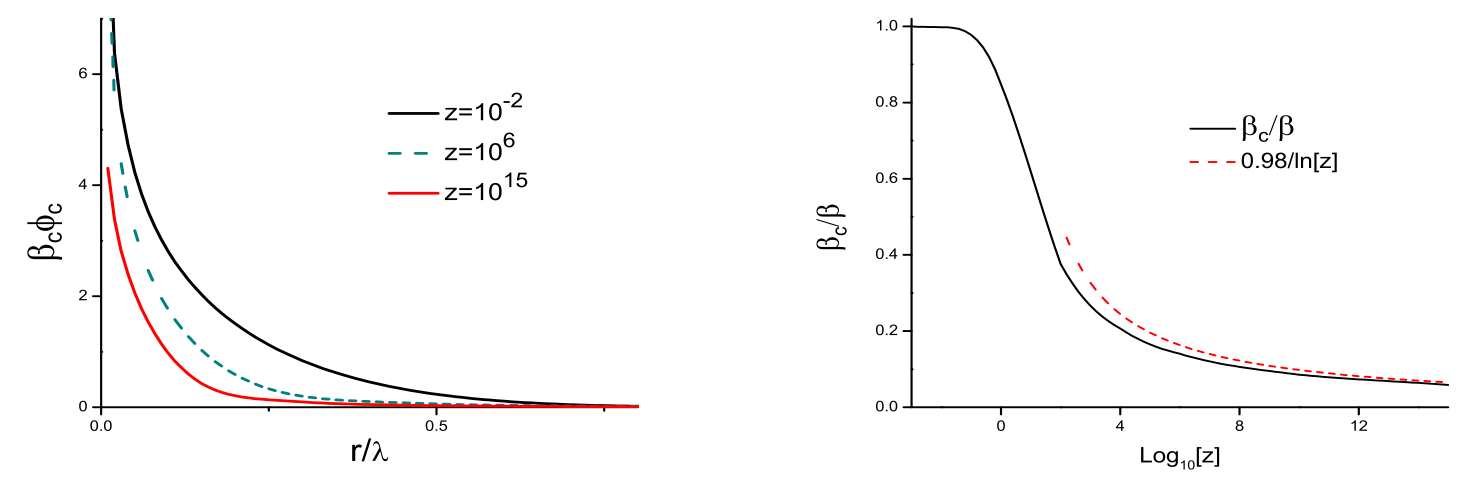

FIG. 1: (a) Dimensionless effective potential at various degeneracies (z values), (b) Effective temperature as a function of z; Asymptotic limit (dotted) implies $T_{c}$ is finite at $T=0$.

approximations. For example a good approximation to $\mathcal{J}(\mathbf{r} \mid n, g)$ for Coulomb systems is the hypernetted chain (HNC) form $\mathcal{J}\left(\mathbf{r}, \mathbf{r}^{\prime} \mid n, g\right) \rightarrow-\ln \left(g\left(\mathbf{r}, \mathbf{r}^{\prime}\right)\right)+g\left(\mathbf{r}, \mathbf{r}^{\prime}\right)-1-c\left(\mathbf{r}, \mathbf{r}^{\prime}\right)$, where $c\left(\mathbf{r}, \mathbf{r}^{\prime}\right)$ is the direct correlation function, defined in terms of $g\left(\mathbf{r}, \mathbf{r}^{\prime}\right)$ by the OrnsteinZernicke equation.

It remains to find an equivalent expression for $\beta_{c}$. This is obtained from the first definition of (41) and the classical virial equation for the pressure, which has the form

$$
\frac{\beta_{c}}{\beta}=\frac{\beta_{c} p_{c}}{\beta p}=\mathcal{K}\left[\beta_{c} \mu_{c}, \beta_{c} \phi_{c}, g_{c}\right] \rightarrow \mathcal{K}\left[\beta_{c} \mu_{c}, \beta_{c} \phi_{c}, g\right]
$$

In the last step $g_{c}$ has been replaced by $g$ according to (6). Since $\beta_{c} \mu_{c}$ and $\beta_{c} \phi_{c}$ are known from (8), $\beta_{c} / \beta$ is given entirely in terms of properties of the quantum system.

\section{EXAMPLE - UNIFORM IDEAL FERMI GAS}

An interesting first illustration of this correspondence of classical and quantum system is the uniform ideal Fermi gas. The quantum description of this non-interacting system with only exchange effects is of course simple to analyze. However, the corresponding classical system is a true many-body problem since those same effects translate into a pair potential among all particles [4]. The full many-body problem must be solved to find that effective potential. Here that problem is addressed in terms of the approximate HNC integral equation. Only the results will be given. In Figure (1a) the classical pair potential is shown as a function of $r / \lambda$ where $\lambda$ is the thermal de Broglie wavelength defined in terms of the 


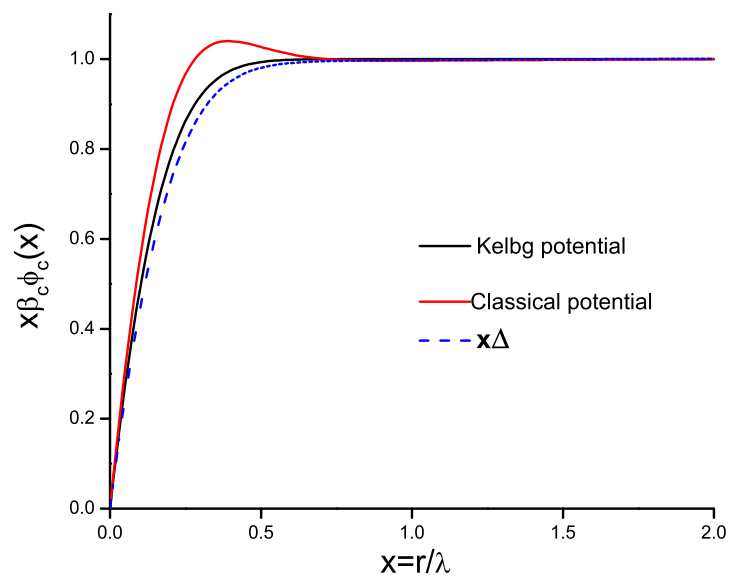

FIG. 2: Classical potential times $r / \lambda$ showing the regularization of Coulomb potential at the origin and comparison with the Kelbg potential.

quantum $\beta$. A family of curves is given for different values of the degeneracy parameter $z=\exp (\beta \mu)$ for the quantum system. Of interest is the development of long range $r^{-2}$ behavior as the temperature is lowered (large z). For this reason the mapping to a classical system requires a uniform neutralizing background to cancel this divergent behavior.

The classical temperature is determined from the virial equation, with $g(r)$ defined as above from the HNC integral equation. This is shown in Figure (1b). As expected for $z<1, \beta_{c} / \beta \sim 1$ while $T_{c}$ approaches a finite value for $T=0$. It is interesting to note that while the pressures of the classical and quantum systems are the same by definition, the internal energies are the same as well. However, this is true only if the internal energy is defined thermodynamically and not mechanically.

\section{ELECTRON GAS}

A practical classical representation for the uniform interacting electron gas can be obtained in a similar way. The classical potential is divided into its ideal gas contribution $\left(\beta_{c} \phi_{c}\right)^{(0)}$ plus a contribution from the Coulomb interactions $\Delta: \beta_{c} \phi_{c}=\left(\beta_{c} \phi_{c}\right)^{(0)}+\Delta$. An approximation for the latter is obtained by requiring the correct RPA form at weak coupling. In the classical context this means that the direct correlation function is given by the classical effective potential. This is obtained from the Ornstein-Zernicke equation with the 
classical $g_{c}(r)$ replaced by the quantum $g(r)$, according to the definition (6), evaluated in the RPA. The final result for the effective classical potential is

$$
\beta_{c} \phi_{c} \rightarrow\left(\beta_{c} \phi_{c}\right)^{(0)}-\frac{1}{n} \int \frac{d \mathbf{k}}{(2 \pi)^{3}} e^{-i \mathbf{k} \cdot \mathbf{r}}\left[\frac{S^{R P A}(k)-1}{S^{R P A}(k)}-\frac{S^{(0)}(k)-1}{S^{(0)}(k)}\right] .
$$

Here $S^{(0)}(k)$ is the ideal gas static structure factor and $S^{R P A}(k)$ is the RPA structure factor. It is possible to show that the familiar Kelbg potential [1] is recovered in the weak coupling, low density limit. Figure (2) illustrates the quantum corrections to the bare Coulomb form for $r_{s}=5$, and $t=T / T_{F}=5$ (where $r_{s}$ is the usual ion sphere radius relative to the Bohr radius, and $T_{F}$ is the Fermi temperature).

\section{ACKNOWLEDGEMENTS}

This research has been supported by NSF/DOE Partnership in Basic Plasma Science and Engineering award DE-FG02-07ER54946 NSF/DOE, and by US DOE Grant de-sc0002139.

[1] A. V. Filinov, V. O. Golubnychiy, M. Bonitz, W. Ebeling, and J. W. Dufty, Phys. Rev. E 70, 046411 (2004).

[2] F. Perrot and M. W. C. Dharma-wardana, Phys. Rev. Lett. 84, 959, 2000; Phys. Rev. B 62, $16536(2000)$.

[3] M. W. C. Dharma-wardana, Int. J. Quant. Chem. (to appear); arXiv:1103.6070.

[4] F. Lado, J. Chem. Phys. 47, 5369 (1967). 